\title{
Apnoeic and obstructive nonapnoeic sleep respiratory events
}

\author{
J.F. Masa***, J. Corral****, J. Teran"***, M.J. Martin*, C. Disdier***, M. Rubio ${ }^{\sharp, * *, ~}$ \\ M. Mota ${ }^{+}$, J. Zamorano ${ }^{\S}$ and J.M. Montserrat ${ }^{f, * *}$
}

ABSTRACT: Obstructive nonapnoeic event (ONE) scoring is shrouded in confusion. This is important in patients with mild disease, in whom precision is crucial.

The aims of the present study were: 1 ) to identify ONEs using oesophageal pressure (OP) (OPONEs) and a noninvasive (NI) method (NI-ONEs); 2) to compare both methods of scoring; and 3) to determine the contribution of ONE definitions to clinical findings.

Patients with suspected sleep apnoeas (respiratory disturbance index $\leqslant 10$ ) during a first polysomnography were subjected to a second with an OP measurement. OP-ONEs and NI-ONEs were defined as an increase in OP or discernible reduction in the amplitude of thoracoabdominal bands with both desaturation and/or arousal. Bland-Altman analysis established agreement. Comparisons were made between OP-ONEs, NI-ONEs and clinical findings.

In our sample $(n=90)$, the addition of an arousal to the NI-ONEs or OP-ONEs with only desaturation increased the number of NI-ONEs by 329 and $362 \%$, respectively. NI-ONEs with arousal and/or desaturation detected $91 \%$ of OP-ONEs. The association with sleepiness depended on the incorporation of arousal into the definition of ONEs.

In patients with mild disease, the addition of an arousal to ONEs, with only desaturation, markedly increased respiratory disturbance index, with probable therapeutic implications. Scoring respiratory events as apnoea and ONEs is easier and sufficiently accurate.

KEYWORDS: Arousal, hypopnoea definition, respiratory effort-related arousal, sleep apnoea syndrome, upper airway resistance syndrome

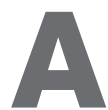
lthough the definition of apnoea is universally accepted, there is considerable uncertainty about the definition of hypopnoea. Despite the recent guidelines from the American Academy of Sleep Medicine (AASM) [1], its definition is based on consensus rather than on data derived from physiological investigations or outcomes. The AASM guidelines provide two rules for the scoring of hypopnoea: 1) a $30 \%$ drop from baseline in the nasal pressure signal excursion (or that of the alternative hypopnoea sensor), with a desaturation of $4 \%$; and 2) a $50 \%$ drop from baseline in the nasal pressure signal excursion (or that of the alternative hypopnoea sensor) with a desaturation of $3 \%$ or an event associated with an arousal. The respiratory effort-related arousal (RERA) is defined as increased respiratory effort or flattening of the nasal pressure waveform, leading to an arousal when the sequence of breaths does not meet the criteria for hypopnoea.

We believe that the aforementioned scoring rules are confused in both physiological and clinical terms. From the physiological point of view, two points should be considered. First, it is difficult to define a quantitative reduction of flow amplitude when using nonquantitative sensors, and secondly, hypopnoeas and RERA are induced by the same pathophysiological process: upper airway dynamic obstruction. Moreover, a further source of confusion arises from the fact that arousal is a hypopnoea and RERA but it is not considered in the case of the first scoring rule for hypopnoea. From the clinical point of view, there are two main considerations. First, the relationship between respiratory events that do not meet the classical criteria for hypopnoea and somnolence; GuilleminAult et al. [2] highlighted the importance of arousals as a cause of somnolence. A thermistor was used as a sensor and this probably accounts for the failure to recognise events noninvasively [3, 4]. Nowadays, other hypopnoea sensors $[5,6]$ can identify the amplitude reduction of the flow signal more successfully. Secondly, the current Medicare guidelines define hypopnoea in terms of desaturation only and do not take into account arousal, as recommended by criterion for both the second scoring rule for
AFFILIATIONS

*Pulmonary Service, San Pedro de Alcántara Hospital,

- Primary Care Center San Jorge,

${ }^{\S}$ Research unit, San Pedro de Alcántara Hospital, Cáceres,

\#Pulmonary Service, General Yagüe, Burgos,

${ }^{+}$Mathematics Dept, Extremadura University, Badajoz,

${ }^{f}$ Hospital Clinic. IDIBAPS, Barcelona, and

${ }^{* *}$ CIBER de enfermedades respiratorias (CIBERES), Spain.

CORRESPONDENCE

J.F. Masa

C/Rafael Alberti 12

10005 Cáceres

Spain

E-mail: fmasa@separ.es

Received:

Oct 232008

Accepted after revision:

Jan 262009

First published online:

Feb 122009

European Respiratory Journal

Print ISSN 0903-1936

Online ISSN 1399-3003 
the second scoring rule for hypopnoea and the scoring rule for RERA. This point is of paramount importance, given that a considerable number of patients with upper airway events and clinical symptoms will not be treated as a result.

Very few papers have addressed this issue to date. CRACOWSKI et al. [7] was one of the first to analyse the problem of studying patients with moderate sleep apnoea. These authors suggested that the distinction between hypopnoea and RERA was artificial. They demonstrated that the level of oesophageal pressure in hypopnoea resembled that of RERA. In the aforementioned study, most of the hypopnoeas displayed arousal without any desaturation. In severe patients, the effect of major hypopnoeas will probably cause marked oxygen desaturation [8-10]. Thus, the addition of an arousal will have less impact in comparison with patients with moderate or mild sleep apnoea, where light or subtle hypopnoeas could be predominant. Given that hypopnoeas and RERA are dependent on the same mechanism, dynamic obstruction, and given that the differences between them could be attributed to the type of flow sensors used, it seems reasonable to score them jointly.

We hypothesise that dividing the respiratory events into apnoeas (static obstruction) and obstructive nonapnoeic events (ONEs; dynamic obstruction) is more representative of the physiopathology of upper airway occlusion and provides the same information as that obtained by taking into account all the different types of nonapnoeic events. Furthermore, in the clinical arena, nonquantitative sensors are routinely used, even though quantitative data are being measured; this discrepancy can lead to errors. Furthermore, the task of subdividing ONES into so many groups is ultimately time-wasting. To test this hypothesis, our aims were as follows: 1) to identify ONEs using oesophageal pressure (OP; OP-ONEs) or a noninvasive method (NI; NI-ONEs); 2) to compare the scoring with the OPONEs versus NI-ONEs definitions; and 3) to evaluate the different ONE definitions with respect to clinical findings.

\section{METHODS}

\section{Study population}

We prospectively analysed a cohort of 90 patients with suspected sleep apnoea syndrome (SAHS) in whom an initial full polysomnography showed an apnoea/hypopnoea index $(\mathrm{AHI}) \leqslant 10$. Apnoeas were defined as an absence of oral-nasal thermistor flow $\geqslant 10 \mathrm{~s}$. Hypopnoea was defined as a discernible reduction in thermistor airflow for $\geqslant 10 \mathrm{~s}$ with $\geqslant 3 \%$ oxygen desaturation or final arousal [11]. Owing to the presence of symptoms, another full polysomnography was performed with oesophageal pressure measurement.

\section{Protocol and measurements}

All subjects were asked to complete the same questionnaire about symptoms of SAHS and other diseases causing sleepiness, and to provide a subjective measurement of sleepiness (Epworth Sleepiness Scale) [12]. The two polysomnographies recorded the electroencephalogram, electro-oculogram, electromyogram, electrocardiogram, oxyhaemoglobin saturation, oral-nasal airflow by thermistor and thoracoabdominal movements by automatically calibrated inductance plethysmography (Respitrace; NIMS, Miami Beach, FL, USA). Sleep data were scored in accordance with the criteria of RECHTSCHAFFEN and KALES [13]. An arousal was defined according to the criteria of the AASM [1]. In the second polysomnography, an additional transnasal oesophageal catheter was introduced and

TABLE 1 Definition of obstructive nonapnoeic events (ONEs) and the respiratory disturbance index (RDI), corresponding to the number of apnoeas and ONEs per hour, based on the type of sensor and their consequences

\section{Event}

OP-ONES

OP-ONEs (desaturation)

OP-ONEs (arousal)

OP-ONEs (desaturation/arousal)

\section{NI-ONES}

NI-ONEs (desaturation)

NI-ONEs (arousal)

NI-ONEs (desaturation/arousal)

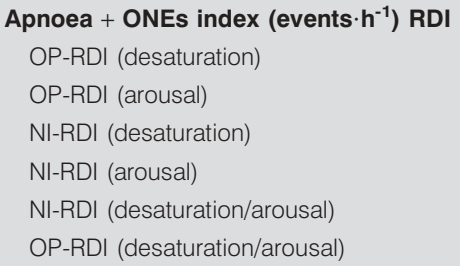

\section{Definition}

A progressive increase in oesophageal pressure for $\geqslant 10 \mathrm{~s}$, followed by $a \geqslant 3 \%$ oxygen desaturation

A progressive increase in oesophageal pressure for $\geqslant 10 \mathrm{~s}$, followed by an arousal

A progressive increase in oesophageal pressure for $\geqslant 10 \mathrm{~s}$, followed by $a \geqslant 3 \%$ oxygen desaturation and/or arousal

A discernible decrease in the thoracoabdominal motion for $\geqslant 10 \mathrm{~s}$, followed by $a \geqslant 3 \%$ oxygen desaturation

A discernible decrease in the thoracoabdominal motion for $\geqslant 10 \mathrm{~s}$, followed by an arousal

A discernible decrease in the thoracoabdominal motion $\geqslant 10 \mathrm{~s}$, followed by $a \geqslant 3 \%$ oxygen desaturation and/or arousal

Number of apnoeas plus OP-ONEs (desaturation)

Number of apnoeas plus OP-ONE (arousal)

Number of apnoeas plus NI-ONEs (desaturation)

Number of apnoeas plus NI-ONEs (arousal)

Number of apnoeas plus NI-ONEs (desaturation/arousal)

Number of apnoeas plus OP-ONEs (desaturation/arousal)

OP: oesophageal pressure; NI: noninvasive. 
calibrated, following the previously described technique [14]. The protocol study was approved by the Institutional Research Committee (Cáceres, Spain) and written consent was obtained from the patients.

As mentioned, the major aim of this paper was to correlate the different variables obtained from the second polysomnography. The role of the first polysomnography was to select patients with probable mild SAHS because a number of patients could have an AHI $>10$ for technical reasons (e.g. underestimation of hypopnoeas by thermistor).

\section{Scoring and definitions in the second polysomnography}

Three different scoring rounds were undertaken. In the first, the oesophageal pressure signal was hidden from the computer screen and the neurological and respiratory variables were assessed, using the same criteria as in the first polysomnography. In the second round, ONEs were scored on the basis of thoracoabdominal bands (NI-ONEs) hiding the oesophageal pressure measurement, and in the third round the score was based on OP (OP-ONEs). We then calculated the different indices, as shown in table 1, according to different definitions of ONEs, as well as their subclasses, based on the OP pressure measurement associated with an arousal and/or a desaturation, and a reduction in the thoracoabdominal motion associated with an arousal and/or a desaturation. Concurrent events (NI-ONEs and OP-ONEs) were not recorded.

\section{Intra- and inter-observer agreement}

In order to evaluate the intra-observer agreement, the technician responsible for the scoring reanalysed the polysomnographies blindly in eight subjects (chosen at random), using the same criteria as in the second and third scoring rounds to identify NI-ONES or OP-ONEs (desaturation/ arousal) and NI-ONES or OP-ONEs (desaturation). Moreover, to evaluate the inter-observer agreement, another technician with similar experience from the same sleep laboratory analysed the same eight subjects twice, using the same protocol. Finally, a researcher (J.F. Masa) examined the agreement by reviewing the same polysomnographic recording. A total of 3,892 epochs of sleep time with oesophageal pressure measurement were checked in each agreement.

\section{Number of RERAs}

The difference between OP-ONEs (arousal) and NI-ONEs (arousal) was considered as the true RERA.

\section{Statistical analysis}

Proportions of patients with sleep apnoea were evaluated at three different OP-RDI and NI-RDI cut-off points $(\geqslant 5, \geqslant 10$ and $\geqslant 15$ ), in the light of the diverse definitions of ONEs.

In order to determine the association between NI-RDI and the Epworth Sleepiness Scale, we compared the mean of NI-RDI (desaturation) and the mean of NI-RDI (desaturation/arousal) in accordance with tertiles of the Epworth Sleepiness Scale. We initially used one-way ANOVA. Where appropriate, differences between individual means were tested using the least significant difference (SPSS 14.0; SPSS Inc., Chicago, IL, USA). If the variables were not distributed normally, a nonparametric test was used (Kruskal-Wallis and Dunn post hoc to identify differences between individual means).

\begin{tabular}{|c|c|c|}
\hline \multirow[t]{2}{*}{ TABLE 2} & \multicolumn{2}{|c|}{$\begin{array}{l}\text { Anthropometric, clinical and polysomnographic } \\
\text { variables from the total sample }\end{array}$} \\
\hline & & Total sample \\
\hline \multicolumn{2}{|l|}{ Subjects n } & 90 \\
\hline \multicolumn{2}{|l|}{ Age yrs } & $44 \pm 10$ \\
\hline \multicolumn{2}{|l|}{ Male sex \% } & 81 \\
\hline \multicolumn{2}{|c|}{ Body mass index $\mathrm{kg} \cdot \mathrm{m}^{-2}$} & $28 \pm 4$ \\
\hline \multicolumn{2}{|c|}{ Habitual snorer \% } & 89 \\
\hline \multicolumn{2}{|c|}{ Apnoeas observed \% } & 54 \\
\hline \multicolumn{2}{|c|}{ Morning fatigue \% } & 61 \\
\hline \multicolumn{2}{|c|}{ Nocturia \% } & 32 \\
\hline \multicolumn{2}{|c|}{ Epworth Sleepiness Scale } & $9 \pm 4$ \\
\hline \multicolumn{2}{|c|}{ Hypertension \% } & 27 \\
\hline \multicolumn{2}{|c|}{ Shift work \% } & 13 \\
\hline \multicolumn{2}{|c|}{$\mathrm{TST}^{\#} \min$} & $262 \pm 62$ \\
\hline \multicolumn{2}{|c|}{ Sleep time lost $\mathrm{min}$} & $28 \pm 60$ \\
\hline \multicolumn{2}{|c|}{ Light sleep \% } & $38 \pm 10$ \\
\hline \multicolumn{2}{|c|}{ Deep sleep \% } & $21 \pm 10$ \\
\hline \multicolumn{2}{|c|}{ REM sleep \% } & $10 \pm 6$ \\
\hline \multicolumn{2}{|c|}{ Arousal index } & $29 \pm 16$ \\
\hline \multicolumn{2}{|c|}{$\mathrm{Sa}, \mathrm{O}_{2}<90 \% \%$ of TST } & $6 \pm 17$ \\
\hline \multicolumn{2}{|c|}{ AHlthermistor } & $3.9 \pm 5.4$ \\
\hline \multicolumn{2}{|c|}{ OP-RDI (desaturation) } & $3.6 \pm 4.7$ \\
\hline \multicolumn{2}{|c|}{ NI-RDI (desaturation) } & $3.3 \pm 4.3$ \\
\hline \multicolumn{2}{|c|}{ NI-RDI (desaturation/arousal) } & $11 \pm 11$ \\
\hline \multicolumn{2}{|c|}{ OP-RDI (desaturation/arousal) } & $13 \pm 13$ \\
\hline
\end{tabular}

Data are presented as mean \pm SD, unless otherwise stated. TST: total sleep time; REM: rapid eye movement; $\mathrm{Sa}_{2} \mathrm{O}_{2}$ : arterial oxygen saturation; AHlthermistor: apnoea/hypopnoea index measured by the thermistor. OP-RDI: apnoeas plus obstructive nonapnoeic events index measuring the obstructive nonapnoeic events (ONEs) by using oesophageal pressure; NI-RDI: apnoeas plus obstructive nonapnoeic events index measuring the ONEs by using bands. ${ }^{*}$ : TST with oesophageal pressure measurement functioning normally; ' time lost caused by poor signal from the oesophageal pressure catheter.

A Bland-Altman analysis was carried out [15] to verify the agreement of the OP-ONE and NI-ONE indexes and to ascertain whether the differences were independent of the measurement size. The analysis was performed with the differences in the OP-ONE (desaturation) and NI-ONE (desaturation) indexes and the mean of the OP-ONE (desaturation) and NI-ONE (desaturation) indexes. Other analyses were carried out on the differences in the OP-ONE (desaturation/arousal) and NI-ONE (desaturation/arousal) indexes and the mean of the OP-ONE (desaturation/arousal) and NI-ONE (desaturation/arousal) indexes.

An event-by-event analysis was performed with the $\kappa$ test $[16,17]$ to determine the intra-observer and inter-observer agreement, excluding the random effects classifying NI-ONEs and OP-ONEs.

\section{RESULTS}

Table 2 shows the anthropometric, clinical and polysomnographic variables of the sample. Patients constituted a middleaged, mainly male and slightly overweight population with 


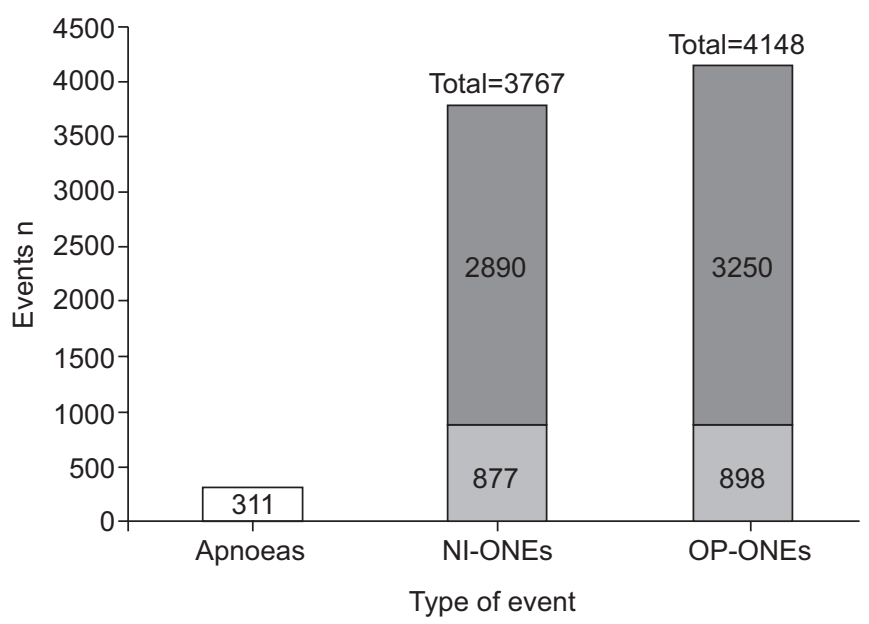

FIGURE 1. Number of respiratory events. Most of the events were OP-ONEs or $\mathrm{NI}$-ONEs with arousal ( $\square$; without oxygen desaturation). $\mathbf{1}$ : desaturation. OPONEs: obstructive nonapnoeic events measured using oesophageal pressure; NIONEs: obstructive nonapnoeic events measured noninvasively using bands.

some clinical and polysomnographic characteristics habitually observed in patients with sleep apnoea.

Table 2 also shows the respiratory events expressed as the apnoea plus ONE index (OP-RDI and NI-RDI), using the different definitions of ONEs. As observed, the OP-RDI (desaturation) and NI-RDI (desaturation) show a 3-4-fold increase when arousal was added to the definition.

Figure 1 shows the data with regard to the number of apnoeas, NI-ONEs and OP-ONEs. The total number of respiratory events identified in the 90 patients studied was: 311 apnoeas, 877 NI-ONEs (desaturation), 898 OP-ONEs (desaturation),

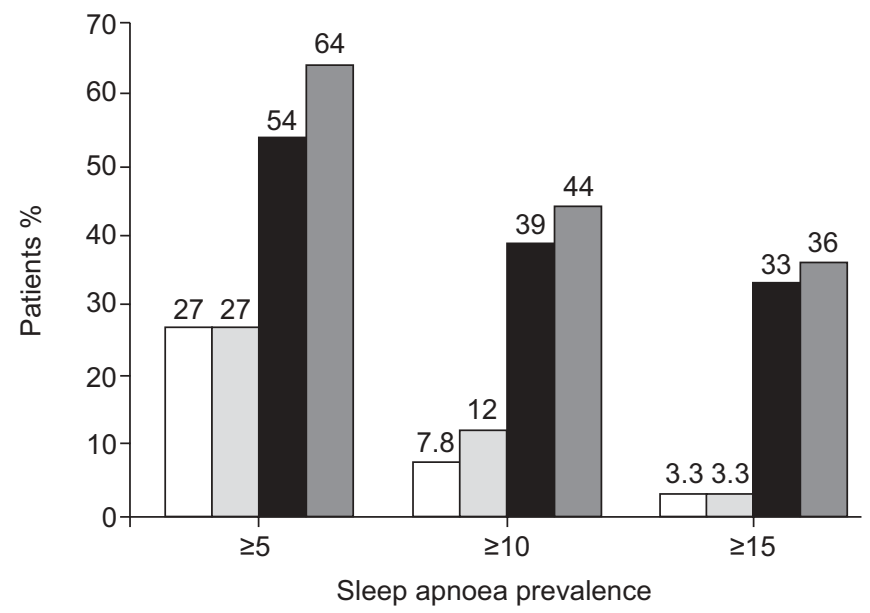

FIGURE 2. Prevalence of sleep apnoea at three cut-off points. The prevalence using OP-RDI (desaturation/arousal)(Ш) or NI-RDI (desaturation/arousal)(匹) was 2-11-times higher that observed when OP-RDI (desaturation)( $\square$ ) or NI-RDI (desaturation)( $\square$ ) were used. Minor differences were observed between OP-RDI (desaturation/arousal) and NI-RDI (desaturation/arousal). OP-RDI: apnoeas plus obstructive nonapnoeic events index measuring the obstructive nonapnoeic events (ONEs) by using oesophageal pressure; NI-RDI: apnoeas plus obstructive nonapnoeic events index measuring the ONEs by using bands.

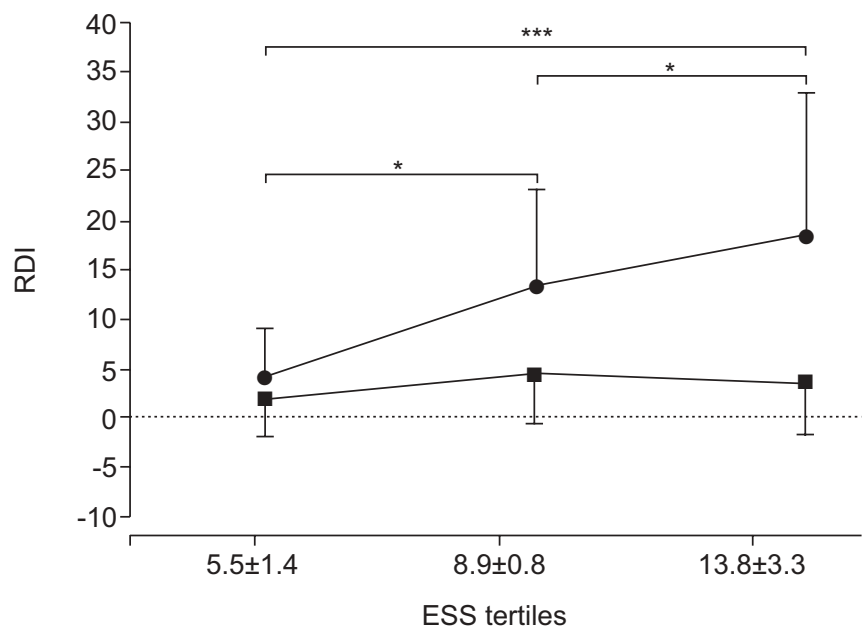

FIGURE 3. Distribution of respiratory disturbance index measured noninvasively (NI-RDI) in accordance with Epworth Sleepiness Scale (ESS) tertiles $(5.5 \pm 1.4, n=34 ; 8.9 \pm 0.8, n=37 ; 13.8 \pm 3.3, n=19)$. In NI-RDI (desaturation/ arousal)(•), all the individual comparisons were statistically significant. In NI-RDI (desaturation)( $\mathbf{\square})$, only the lowest versus intermediate tertiles were statistically significant. ${ }^{*}: p<0.05 ;{ }^{* *}: p<0.001$.

2,890 NI-ONEs (arousals) and 3,250 OP-ONEs (arousals). The sum of the NI-ONEs (desaturation) plus those with arousal, NI-ONEs (desaturation/arousal), was 3,767, whereas the sum of OP-ONEs (desaturation) plus those with arousal, OP-ONEs (desaturation/arousal), was 4,148. In summary, if we add the arousal to NI-ONEs (desaturation) and OP-ONEs (desaturation), the number of NI-ONEs increases by $329 \%$ and by $362 \%$, respectively. Therefore, the number of true RERAs would be the difference between 3,250 OP-ONEs (arousals) and 2,890 NIONEs (arousals). This means that $9 \%$ of the classic RERAs are not detected by our definition of NI-ONEs.

Figure 2 shows the prevalence of sleep apnoea by means of three cut-off points of RDI. The prevalence using OP-RDI or NI-RDI (desaturation/arousal) was 2-11-times higher than that observed when only OP-RDI or NI-RDI (desaturation) was used.

Figure 3 shows that there was no increase in NI-RDI (desaturation) according to the Epworth Sleepiness Scale tertiles. However, the association was significant $(p<0.001)$ when arousal was included in the definition of ONE, with NIRDI (desaturation/arousal). Furthermore the Epworth sleepiness scale was significantly correlated with the arousal index $(\mathrm{r}=0.373, \mathrm{p}<0.001)$. This could lead to an increase in the number of patients in need of treatment.

Figure 4 shows the Bland-Altman analysis between: OP-ONE index (desaturation) and NI-ONE index (desaturation) (fig. 4a); OP-ONE index (desaturation/arousal) and NI-ONE index (desaturation/arousal) (fig. 4b). The difference between the OP-ONE-index (desaturation) and the NI-ONE-index (desaturation) was $0.4 \pm 1$. The difference between OP-ONEindex (desaturation/arousal) and the NI-ONE-index (desaturation/arousal) was $1.9 \pm 2.8$. The difference between the OPONE-index and NI-ONE-index was independent of the measurement size. 

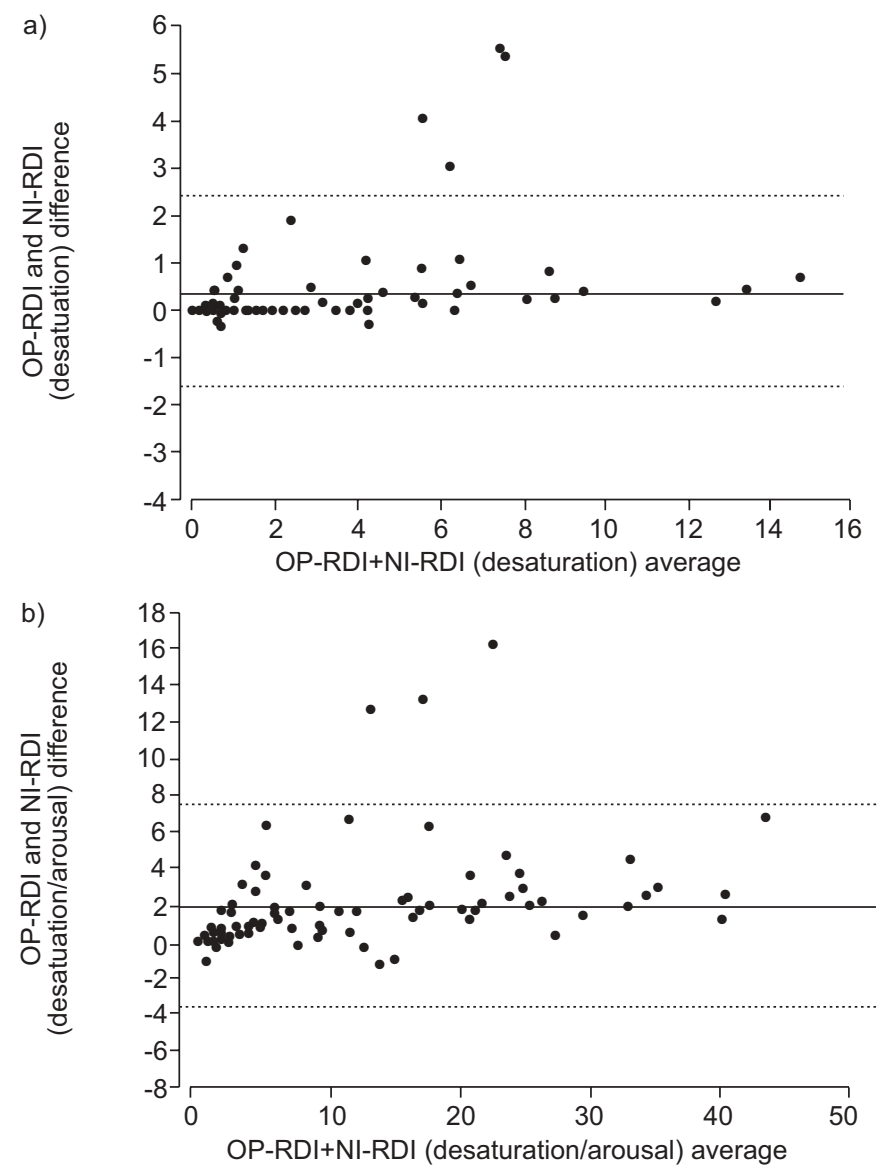

FIGURE 4. Bland-Altman analysis between a) OP-ONE index (desaturation) and NI-ONE index (desaturation) and b) OP-ONE index (desaturation/arousal) and NI-ONE index (desaturation/arousal) (bottom). Minor differences were found in both the analyses, regardless of the measurement size. OP-RDI: apnoeas plus obstructive nonapnoeic events index measuring the obstructive nonapnoeic events (ONEs) by using oesophageal pressure; NI-RDI: apnoeas plus obstructive nonapnoeic events index measuring the ONEs by using bands.

Finally, the intra- and inter-observer agreement: the intraobserver agreement ( $\kappa$ test) was 0.82 for NP-ONEs (desaturation/arousal) and 0.93 for OP-ONEs (desaturation/arousal). The inter-observer agreement was 0.86 for NI-ONEs (desaturation/arousal) and 0.90 for OP-ONEs (desaturation/arousal). The intra-observer agreement was 0.87 for NP-ONEs (desaturation) and 0.96 for OP-ONEs (desaturation). The interobserver agreement was 0.90 for NI-ONEs (desaturation) and 0.94 for OP-ONEs (desaturation).

\section{DISCUSSION}

This study reviews a large number of ONEs and shows that, in our selected population, arousal plays the major role in RDIs, especially in more symptomatic patients. Furthermore, the use of OP instead of bands for the ONE classification increases the number of RDIs by only $9 \%$. In the highest Epworth Sleepiness Scale tertile (13.8 \pm 3.2$)$, the NI-RDI changed from $3.6 \pm 4.9$ with only desaturation to $18.8 \pm 14.9$ with desaturation and/or arousal. This finding could have therapeutic implications.

The ONE definition proposed (discernible reduction in the band amplitude with arousal and/or fall in arterial oxygen saturation $\left.\left(\mathrm{Sa}_{2} \mathrm{O}_{2}\right)\right)$ is more suitable than those proposed by the AASM, given that the majority of the sensors used in clinical practice are nonquantitative (AASM definitions quantify the degree of flow reduction), and most ONEs are associated with arousal alone (77\%) (the first AASM hypopnoea definition does not include arousal). It is therefore reasonable to assume that the recommended AASM hypopnoea definition does not embrace many obstructive events.

CRACOWSKI et al. [7] carried out a study with a similar objective and with similar criteria for patient selection. Moreover, they found that $63 \%$ of their 1,061 OP-ONEs had cortical arousal with no oxygen desaturation. Other authors, however, have not obtained similar results [8-10]. These discrepancies could be attributed to varying definitions of hypopnoea or differences in the sensors or criteria for patient selection. In our study, as in that of CRACOWSKI et al. [7], the subjects were selected on the basis of suspected moderate-mild sleep apnoea and a limited number of oxygen desaturations. In contrast to typical sleep apnoea patients [8-10], CRACOWSKI et al. [7] and the present study showed a low body mass index (BMI) $[8,9]$. A higher BMI or greater breathing reduction could induce more oxygen desaturation, thereby reducing the contribution of arousal to hypopnoea detection.

The standard AASM hypopnoea definition does not include arousal, largely because of a study that demonstrated the greater reliability of desaturation [18]. The intra-class correlation coefficient was 0.97 considering RDI with desaturation $\geqslant 3 \%$ alone, 0.77 considering arousal alone and 0.95 considering desaturation $\geqslant 3 \%$ or arousal. Our more powerful eventby-event analysis ( $\kappa$ test) revealed the same phenomenon, but with a lower magnitude. As indicated previously, our explanation is that desaturation habitually coincides with a greater reduction in breathing, in which case it is easy to recognise; ONEs with arousal alone coincide more frequently with a lower reduction in breathing and are more difficult to identify. The reliability level for desaturation/arousal was very good, which means that this method can be applied in clinical practice.

Although oesophageal pressure is the standard for measuring respiratory effort [19], it is not able to distinguish between hypopnoeas and RERAs. When considering our definition of NI-ONE as discernible reductions in thoracic-abdominal bands plus desaturation and/or arousal, only $9 \%$ of the OP-ONEs were undetected. Accordingly, we regarded this group as true RERAs. Similar results were found in CRACOWSKI et al. [7] study, where the RERAs accounted for only $5 \%$ of the total ONEs when the flow was measured by pneumotacography and the effort by OP. Thus, the number of ONEs identified in the present study resembles the level achieved in the current practice of polysomnography, where slight reductions in the flow measurement are taken into account. Given that RERAs and hypopnoeas have the same physiopathological aetiology and even similar clinical symptoms, it may be assumed that the definitions of these events overlap. The scoring of the two events should therefore be performed jointly in order to simplify routine practice.

The sleep apnoea prevalence increased 2-11-times more when arousal was considered in the ONE definition (fig. 2). 
Differences in prevalence between noninvasive and invasive methods (NI-RDI and OP-RDI) were small and were mainly found when desaturation/arousal criteria were applied. These differences were higher in the lower cut-off points of diagnosis. We believe that this is probably explained by the detection of RERA events by the invasive method.

One limitation of the present study is that we did not use nasal pressure as the flow sensor to score the number of hypopnoeas and RERAs. Instead, we used thoracoabdominal bands (inductance plethysmography), regarded as an alternative hypopnoea measurement [1, 20]. Bands calibrated with a known volume have been considered appropriate for obtaining breath volume [21] with a good agreement with nasal pressure [22, 23]. We did not calibrate the inductance plethysmography device with a known volume but instead used autocalibration. We found that inductive plethysmography satisfactorily identified ONEs when compared with the standard respiratory effort measurement (OP). Accordingly, similar results would be expected when using nasal pressure. However, it could be possible that the amount of RERAs detected would be slightly higher with nasal prongs measurements, reducing our $9 \%$ of undetected RERAs.

Thus, arousal plays a major role in the detection of respiratory events in mild sleep apnoea patients, and this could have therapeutic implications. Arousal should therefore always be added to the recommended AASM hypopnoea definition, in order to enhance the evaluation of this population. If scoring were based on a discernible reduction in the amplitude of the breathing signal with arousal and/or desaturation, almost all respiratory events (hyponeas and RERAs) would be detected. We suggest that nonapnoeic respiratory events should therefore be scored jointly in order to simplify routine practice.

\section{SUPPORT STATEMENT}

Supported by SEAR (Sociedad Extremeña del Aparato Respiratorio; Extremadura, Spain) and ANCA (Asociación de Neumólogos de Cáceres; Extremadura, Spain).

\section{STATEMENT OF INTEREST}

None declared.

\section{ACKNOWLEDGEMENTS}

We are indebted to V. Rodríguez (Consejería de Educación, Junta de Extremadura, Spain) and V. Iglesias (San Pedro de Alcántara Hospital, Cáceres, Spain) for assistance in the translation of the manuscript and A. Martín and C. Lorenzana (both San Pedro de Alcántara Hospital, Cáceres, Spain) for technical assistance.

\section{REFERENCES}

1 American Academy of Sleep medicine (AASM). International Classification of Iber C. S. Ancoli-Israel, AL Chesson, SF Quan. The AASM Manual for the Scoring of Sleep and Associated Events. 2007.

2 Guilleminault C, Stoohs R, Clerck A, et al. Cause of excessive daytime sleepiness. The upper airway resistance syndrome. Chest 1993; 104: 781-787.
3 Berg S, Haight JS, Yap V, et al. Comparison of direct and indirect measurements of respiratory airflow: implications for hypopneas. Sleep 1997; 20: 60-64.

4 Farré R, Montserrat JM, Rotger M, et al. Accuracy of thermistors and termocouples as flow-measuring devices for detecting hypopneas. Eur Respir J 1998; 11: 197-182.

5 Ayappa I, Norman RG, Krieger AC, et al. Non-invasive detection of respiratory effort-related arousals (RERAs) by a nasal cannula/ pressure transducer system. Sleep 2000; 23: 763-771.

6 Masa JF, Corral J, Martín MJ, et al. Assessment of thoracoabdominal bands to detect respiratory effort-related arousal. Eur Respir J 2003; 22: 661-667.

7 Cracowski C, Pépin JL, Wuyam B, et al. Characterization of obstructive nonapneic respiratory events in moderate sleep apnea syndrome. Am J Respir Crit Care Med 2001; 164, 6: 944-948.

8 Tsai WH, Flemons WW, Whitelaw WA, Remmers JE. A comparison of apnea-hypopnea indices derived from different definitions of hypopnea. Am J Respir Crit Care Med 1999; 159: 43-48.

9 Manser RL, Rochford P, Pierce RJ, et al. Impact of different criteria for defining hypopneas in the apnea-hypopnea index. Chest 2001; 120: 909-914.

10 Redline S, Kapur VK, Sanders MH, et al. Effect of varying approaches for identifying respiratory disturbances on sleep apnea assessment. Am J Respir Crit Care Med 2000; 161: 369-374.

11 Barbé F, Amilibia J, Capote F, et al. [Diagnosis of obstructive sleep apnea syndrome. Consensus report from the Respiratory Insufficiency and Sleep Disorders Group.] Arch Bronconeumol 1995; 31: 460-462.

12 Johns MW. Daytime sleepiness, snoring and obstructive sleep apnea: the Epworth Sleepiness Scale. Chest 1993; 103: 30-36.

13 Rechtschaffen A, Kales A, eds, Manual of standardized terminology, techniques and scoring system for sleep stages of human subjects. Washington, U.S. Governments Printing Office; 1998.

14 Baydur A, Behrasks PK, Zin WA, et al. A simple method for assessing the validity of the esophageal balloon technique. Am Rev Respir Dis 1982; 126: 788-791.

15 Bland JM, Altman DG. Statistical methods for assessing agreement between two methods of clinical measurement. Lancet 1986; 1 : 307-310.

16 Cohen JA. Coefficient of agreement between nominal scales. Educ Psychol Meas 1960; 20: 37-46.

17 Fleiss JL. Statistical methods for rates and proportion, 2nd Edn. New York, Wiley, 1981; pp. 216-219.

18 Whitney CW, Gottlieb DJ, Redline S, et al. Reliability of scoring respiratory disturbance indices and sleep staging. Sleep 1998; 21: $749-757$.

19 Sleep-related breathing disorders in adults, recommendations for syndrome definition and measurement techniques in clinical research. The Report of an American Academy of Sleep Medicine Task Force. Sleep 1999; 22: 667-689.

20 Kushida CA, Littner MR, Morgenthaler T, et al. Practice parameters for the indications for polysomnography and related procedures: an update for 2005. Sleep 2005; 28: 499-521.

21 Cantineau JP, Escourrou P, Sartene R, et al. Accuracy of respiratory inductive plethysmography during wakefulness and sleep in patients with obstructive sleep apnea. Chest 1992; 102: 1145-1151.

22 Berg S, Haight JS, Yap V, et al. Comparison of direct and indirect measurements of respiratory airflow: implications for hypopneas. Sleep 1997; 20: 60-64.

23 Thurnheer R, Xie X, Bloch KE. Accuracy of nasal cannula pressure recordings for assessment of ventilation during sleep. Am J Respir Crit Care Med 2001; 164: 1914-1919. 